\title{
Endoscopic Skull Base and Transoral Surgery During the COVID-19 Pandemic: Minimizing Droplet Spread with a Negative-Pressure Otolaryngology Viral Isolation Drape (NOVID)
}

\author{
Ivan H. El-Sayed, $\mathrm{MD}^{1}$ \\ ${ }^{1}$ University of California, San Francisco
}

April 27, 2020

\begin{abstract}
Background: The COVID-19 pandemic has raised concern of transmission of infectious organisms through aerosols formation in endonasal and transoral surgery.

Methods: Retrospective review. We introduce the Negative-Pressure Otolaryngology Viral Isolation Drape (NOVID) system to reduce the risk of aerosol. NOVID consists of a plastic drape suspended above the patient's head and surgical field with a smoke evacuator suction placed inside the chamber.

Results: Four patients underwent endonasal (4) and endo-oral surgery (1). Fluorescein was applied to the surgical field. Black light examination of fluorescein treated operative fields revealed minimal contamination distant to the surgical field. In two prolonged cases with high-speed drilling, droplets were identified under the barrier and on the tip of the smoke evacuator. Instruments and cottonoids appeared to be a greater contributor to field contamination.

Conclusions: Negative-pressure aspiration of air under a chamber barrier which appears to successfully keep aerosol and droplet contamination to a minimum.
\end{abstract}

\section{Abel P. David, MD ${ }^{1}$; Nicole T. Jiam, MD ${ }^{1}$; Joshua M. Reither, CST ${ }^{2,3}$; Jose G. Gurrola II, MD ${ }^{1,2}$; Manish Aghi, MD PhD ${ }^{2,4}$; Ivan H. El-Sayed, $\mathrm{MD}^{1,2}$}

${ }^{1}$ Department of Otolaryngology-Head \& Neck Surgery, University of California, San Francisco (San Francisco, CA)

${ }^{2}$ Center for Minimally Invasive Skull Base Surgery, University of California, San Francisco (San Francisco, CA)

${ }^{3}$ University of California, San Francisco Medical Center (San Francisco, CA)

${ }^{4}$ Department of Neurological Surgery, University of California, San Francisco (San Francisco, CA)

\section{Corresponding Author:}

Ivan H. El-Sayed, MD

Department of Otolaryngology-Head and Neck Surgery, University of California, San Francisco

2233 Post Street, UCSF Box 349, San Francisco, California 94115

Phone: 415-353-2757

E-mail: Ivan.El-Sayed@ucsf.edu 


\title{
Disclosure of Funding: None
}

Short Running Head: Preventing Droplet Spread During Skull Base Surgery

Keywords: COVID-19; aerosolization; skull base; endoscopic surgery; negative-pressure

\begin{abstract}
Background: The COVID-19 pandemic has raised concern of transmission of infectious organisms through aerosols formation in endonasal and transoral surgery.

Methods: Retrospective review. We introduce the Negative-Pressure Otolaryngology Viral Isolation Drape (NOVID) system to reduce the risk of aerosol. NOVID consists of a plastic drape suspended above the patient's head and surgical field with a smoke evacuator suction placed inside the chamber.

Results: Four patients underwent endonasal (4) and endo-oral surgery (1). Fluorescein was applied to the surgical field. Black light examination of fluorescein treated operative fields revealed minimal contamination distant to the surgical field. In two prolonged cases with high-speed drilling, droplets were identified under the barrier and on the tip of the smoke evacuator. Instruments and cottonoids appeared to be a greater contributor to field contamination.
\end{abstract}

Conclusions: Negative-pressure aspiration of air under a chamber barrier which appears to successfully keep aerosol and droplet contamination to a minimum.

\section{Introduction}

The novel coronavirus (SARS-CoV-2) is a highly-transmissible zoonotic respiratory virus with an astonishing global impact. ${ }^{1}$ In December 2019, the first cases emerged in Wuhan, China ${ }^{2}$, and on March 11, 2020 the novel coronavirus disease (COVID-19) was declared a worldwide pandemic by the World Health Organization (WHO). During this pandemic, patients continue to present with urgent head and neck, and skull base conditions requiring timely surgery. Strategies to reduce risk of spread of infection during surgery are needed to protect the surgical team and patients.

While many infected with SARS-CoV2 will be asymptomatic, there are those with COVID-19 who will develop an advanced form of the disease presenting as an atypical pneumonia requiring ventilatory support and associated with significant morbidity and mortality. ${ }^{1}$ It is known that there is a high viral load in the nasal cavity, nasopharynx, and oropharynx; the only higher subsite being the lower respiratory tract. ${ }^{3}$ What is more worrisome, is that infected asymptomatic carriers carry the same high viral load in these anatomic sites as those with symptoms. ${ }^{4,5}$ Among healthcare workers, certain personal protective precautions are taken, particularly during aerosol generating procedures, but these are not guaranteed to work. ${ }^{6}$

At the onset of the pandemic, anecdotal international reports suggested otolaryngologists are also placed at great risk during surgery. ${ }^{7}$ Intuitively, surgery of the aerodigestive tract can expose workers to infected fluids and the use of high-speed drills in these anatomic sites can be a contributing factor. Workman et al. demonstrated in a simulated skull base surgery scenario that high-speed drills generated aerosols and droplet contamination up to $36 \mathrm{~cm}$ away from the patient. ${ }^{8}$ Coronaviruses are well studied, and measure approximately 0.125 microns and are transported in respiratory droplets. ${ }^{9}$ Coronaviruses can remain aerosolized for up to 3 hours, and are stable on surfaces like plastic and stainless steel. ${ }^{10}$ Improved methods for preventing droplet and aerosol spread are needed especially during high-risk procedures. Here we describe our experience developing the Negative-Pressure Otolaryngology Viral Isolation Drape (NOVID) to reduce aerosol and droplet spread in and around the surgical field.

\section{Methods}


This is a retrospective descriptive review of our technique used in four patients who underwent endonasal and/or endo-oral surgery during the COVID-19 pandemic, in which a negative-pressure chamber barrier was installed around the surgical field.

\section{Protective Barrier and Negative Pressure Chamber}

We constructed a chamber around the patients head which consisted of a transparent plastic film used to cover a patient's head. The plastic film was a $112 \mathrm{~cm}$ x $112 \mathrm{~cm}$ fluid warmer drape (Ecolab; St. Paul, MN) placed over a Lone Star disposable ring retractor (Cooper Surgical; Product \#3308; Trumbull, CT) that was suspended by a Bookwalter Retractor Laparoscopic Support Set (Symmetry Surgical; Product \#50-5730; Nashville, TN) (Figure 1A). A space between the patients head and the barrier was created to prevent the drape from being pulled into the suction port (Figure 1B). Non-penetrating towel clamps were used to secure the plastic film to the Lone Star and the bed to keep the drape taught over the surgical field. Fenestrations were created in the plastic barrier drape to allow passage of instruments for either an endonasal or endo-oral procedure (Figure 1C). These materials were readily available at our institution and are likely used by most general surgeons performing laparoscopic abdominal surgery.

\section{Negative Pressure Aerosol Chamber}

Smoke evacuator suction tubing (Stryker Corporation; Product\# 0700-026-000; Kalamazoo, MI) was placed under the drape and connected to a Neptune 3 Waste Management System (Stryker Corporation; Kalamazoo, MI) to create a negative pressure chamber for the aerosol housing system. The Neptune 3 Waste Management System is a surgical suction system designed to provide both hazardous fluid and smoke evacuation during surgery. It contains both a smoke evacuator filter compartment with an ultra-low penetrating air (ULPA) efficiency rating and a fluid suction high-efficiency particulate air (HEPA) filter compartment. The tubing is connected to the smoke evacuator manifold port on the Neptune suction system to provide a minimum negative pressure of 0.5 inches of water gauge. These metrics are consistent with the minimum ventilation requirements for Class III biological safety cabinets in California. In addition to the suspension apparatus, we created one or more ventilation ports, located near head of the bed, to allow airflow into the negativepressure chamber. We were not able to measure the pressure under the drape but did verify the suction was active, and when these openings for air intake were occluded the drape tended to collapse.

In the first case and NOVID design, a polyethylene film drape (3M Steri Drape 1010) was supported only by a straight Bookwalter bar. Unfortunately, the single bar did not provide enough structural support, and the drape collapsed and occlude the smoke evacuator under vacuum limiting aerosol clearance. In the final iteration of the negative pressure housing system, we used the Lone Star ring retractor attached to a laparoscopic Bookwalter retractor holder to suspend the drape above the patient's face without collapse when the negative pressure environment was created. Gauze was also placed near the tip of the smoke evacuator to prevent the drape from occluding the smoke evacuator tip during the surgery.

\section{Fluorescein Markers for Droplet Spread}

We used fluorescein dye and ultraviolet (UV) light to assess the efficacy of the negative pressure housing system in minimizing aerosol dispersion. Droplet formation and patient secretion spread was evaluated with the application of fluorescein into the nasal cavity. We diluted fluorescein (Fluorescite, fluorescein injection USP, $10 \%$ at $100 \mathrm{mg} / \mathrm{ml}$; Alcon Laboratories; Fort Worth, TX) to a concentration of $1 \%$ fluorescein in $10 \mathrm{ml}$ of normal saline solution and topically applied dropwise to the nasal cavity and nasopharynx at the beginning of the case. This was further diluted with saline during the case. The fluorescein solution washes away easily with saline irrigation, so regular reapplication of fluorescein dye was performed the nasal cavity prior to use of instruments like the microdebrider, bipolar and monopolar cautery and high-speed drilling. At the conclusion of the surgery, an evaluation of the surgical drapes and gowns was performed using an ultraviolet Wood's lamp.

We did not have a control group because routine preoperative SARS-CoV-2 testing is not being performed at our institution at this time. As such, a universal personal protective equipment (PPE) policy was applied 
for all aerosol generating surgeries, including endoscopic endonasal and endo-oral surgery, consisting of an N95 mask or powered air-purifying respirator (PAPR), eye protection, and fluid resistant gown.

\section{Additional Surgical Set-Up Considerations}

A stereotactic navigation system (Brainlab; Munich, Germany) was during each endoscopic endonasal surgery. The protective barrier was placed over the reference array without interfering with the infrared tracking. Should it be needed, the reference array can be brought outside the drape through holes in the barrier drape.

\section{Results}

\section{Surgical Cases}

Four patients underwent endoscopic endonasal surgery for osteomyelitis, sinonasal malignancies, nasopharyngeal malignancy, and a pituitary adenoma threatening vision. Two patients required concurrent endoscopic sinus surgery as well. Power instruments with aerosol generating potential were used in these surgeries and are summarized in Table 1. These instruments include a microdebrider (used in 3 cases), electrocautery (used in 3 cases), and a high-speed drill (used in 2 cases).

\section{Black Light Examination}

Our cases demonstrated there is minimal spread of fluorescein beyond the immediate surgical field. We examined the surgical field and adjacent area prior to and after the case with a UV emitting black light (Figure 2). We marked the areas of contamination. In general, we found very little contamination of droplets on the patient. In all cases, we found fluorescent dye at sites round the nares, on the wipe placed on the patient's chest, and on the surgical instrument table. In the first NOVID design, without the Lone Star ring retractor, we found one single droplet on the vertical drape "wall" placed by the anesthesiologist at the foot of the patient. This was about 4 feet from the surgeon. In the two functional endoscopic sinus surgery cases, we did not find droplets under the barrier beyond the nares or near the smoke evacuator. In the transsphenoidal pituitary case, we did identify fluid under the barrier several centimeters away from the nares. In both skull base cases, we identified dye on the gauze placed over the tip of the smoke evacuator (Figure 1D).

Examination of the surgeon revealed a few droplets along the abdomen region and in one case (Table 1, Case 3 ) a single droplet on the surgeon's arm. Also, during this case, the scrub nurse had large droplets $>5 \mathrm{~mm}$ on the abdomen region. However, the nurse was also handling fluorescein stained epinephrine cottonoids and caused self-contamination had self-contaminated. It is noted that instruments from the case are used to pick up cottonoids, such as Bayonet forceps, should be considered contaminated as well.

\section{Discussion}

We present our experience after the introduction of a technique using negative pressure in a housing barrier (NOVID) to reduce the spread of droplets and aerosol during endonasal skull base and transoral surgery. We have used this technique both in the sinonasal region for ethmoidectomy, maxillary antrostomy, transpterygoid nasopharyngectomy, and transsphenoidal sellar skull base surgery, as well as for endo-oral palate surgery. This technique is still in evolution, but we found a minimum of contamination of the surgical field with dye or visible fluid. While the fluorescein is only a surrogate marker, since it is applied only intermittently during the case. Notably, we identified dye at the inlet of the smoke evacuator after the skull base procedures, indicating it is capturing fluid in the form of droplets or aerosol under NOVID.

A surgeon routinely places suction in the patient's nasal cavity. However, the routine suction is attached to the HEPA filter in the Neptune suction machine which captures particles down to 0.3 microns. The smoke evacuator ULPA filter attachment on the side of the Neptune captures particles down to 0.1 microns. ${ }^{11}$ The COVID-19 virus is 0.125 microns and in theory should be captured by an ULPA filter but not the standard suction. $^{9}$ Viral particles could theoretically escape from the Neptune system with the standard filter. We 
did examine the Neptune machine at the end of the surgery and found the fluid in the container to brightly fluoresce, and no dye visible outside the machine.

In general, we found droplet spread where the instruments or cottonoids were placed. We did find droplets on the surgeon's abdomen and on the surgeon's arm. One droplet was found at the foot of the bed. In the two cases that generated aerosol we found the droplets under the drape away from the nares. Whether this occurred due to aerosol from drilling or due to irrigation and instrument passing over a prolonged time period, we cannot determine at this time. However, in general we found very little droplet contamination away from the surgical field. Large droplets were found at expected locations: the patient's chest where the gauze wipe is located, the instrument table, around the epinephrine cottonoids, and in one case on the nurse's abdomen. This suggest that surgical fomites including the instruments, cottonoids and tissue specimens are a major source of spread of fluid to the surgical field. We suspect the droplet at the patient's foot occurred during removal of an instrument or cottonoid as it was in a linear trajectory and no other droplets identified outside the surgical field. This suggests that methods to reduce contamination caused by surgical instruments and cottonoids may be useful. We suggest, gentle withdrawal of instruments from the nasal cavity and passage of cottonoids and biopsy specimens off the field onto a plastic tray similar to the plastic safe sharps passing tray used for the hands-free transfer or sharps in many operating rooms. ${ }^{12}$

There is wide concern within the skull base field regarding aerosol generation and droplets spread during endoscopic endonasal and upper aerodigestive tract surgery. The NOVID system combines a fluid resistant barrier and a negative pressure environment to reduce the spread of aerosols and droplets. One alternate option is to place a suction into the nasal cavity to create negative pressure environment. ${ }^{8}$ However, this may obstruct access to the surgical field or become clogged. Surgeons commonly use suctions while operating and drilling, but they can also become clogged, or be too deep in the field to catch the aerosol generated anteriorly. NOVID also allowed for repetitive entry and removal of instruments into and out of the surgical field and allows the surgeon to operate unencumbered. There are potential improvements in the instrument port design. We envision that the ideal surgical port would create a better seal around the instrument and could wipe off any fluid or droplet contamination from the scope and instruments as it is removed from the barrier.

NOVID also appears to reduce splash back during irrigation, and the negative pressure may capture aerosol created by cauterization or high-speed drilling. Identification of fluorescence at the smoke evacuator tip suggests it was able to draw aerosolized droplets. It is a limitation of this study that due to the urgent concerns created during this pandemic, we do not have control group to compare droplet spread. Nevertheless, it is encouraging to find in the skull base cases, no droplets or fluorescence on the outside of the drape or around the patient's head other than where the instruments were direct contact. Also, fluorescein is routinely used on the epinephrine soaked cottonoids, which could cause false positive findings. However, the cottonoids after being removed from the patient should be considered contaminated.

We also found the nursing staff and anesthesia team to be extremely appreciative of the monitoring of spread of patient's fluids into the operating room environment and with the use of a barrier. This assessment seemed to provide the team some peace of mind to see how, where, and the minimal amount of contamination that occurred. We now routinely use the fluorescein during each case as a marker of fluid spread for quality assurance for all the operating room staff.

\section{Conclusion}

Reducing fluid spread during surgery involving the upper aerodigestive tract in the COVID-19 era is important for the positive or indeterminate patient. We introduce the use of a negative pressure system which may be more efficacious than a simple barrier in minimizing droplet contamination. Our preliminary findings indicate regional containment of the fluorescein dye within the housing system and at the smoke evacuator port. We also identified other potential sources of field contamination from surgical fomites suggests gentle instrument movements and isolation of materials removed from the nasal cavity are important.

\section{References}


1. Zhou F, Yu T, Du R, et al. Clinical course and risk factors for mortality of adult inpatients with COVID-19 in Wuhan, China: a retrospective cohort study. Lancet. 2020;395(10229):1054-1062.

2. Huang C, Wang Y, Li X, et al. Clinical features of patients infected with 2019 novel coronavirus in Wuhan, China. Lancet.2020;395(10223):497-506.

3. Wang W, Xu Y, Gao R, et al. Detection of SARS-CoV-2 in Different Types of Clinical Specimens. JAMA. 2020 .

4. Rothe C, Schunk M, Sothmann P, et al. Transmission of 2019-nCoV Infection from an Asymptomatic Contact in Germany. N Engl J Med.2020;382(10):970-971.

5. Zou L, Ruan F, Huang M, et al. SARS-CoV-2 Viral Load in Upper Respiratory Specimens of Infected Patients. N Engl J Med.2020;382(12):1177-1179.

6. Tran K, Cimon K, Severn M, Pessoa-Silva CL, Conly J. Aerosol generating procedures and risk of transmission of acute respiratory infections to healthcare workers: a systematic review. PLoS One.2012;7(4):e35797.

7. Vukkadala N, Qian ZJ, Holsinger FC, Patel ZM, Rosenthal E. COVID-19 and the otolaryngologist preliminary evidence-based review.Laryngoscope. 2020.

8. Workman AD, Welling DB, Carter BS, et al. Endonasal instrumentation and aerosolization risk in the era of COVID-19: simulation, literature review, and proposed mitigation strategies. Int Forum Allergy Rhinol. 2020 .

9. Fehr AR, Perlman S. Coronaviruses: an overview of their replication and pathogenesis. Methods Mol Biol. 2015;1282:1-23.

10. van Doremalen N, Bushmaker T, Morris DH, et al. Aerosol and Surface Stability of SARS-CoV-2 as Compared with SARS-CoV-1. N Engl J Med. 2020.

11. Neptune 3: Waste Management System Smoke Evacuator. https://www.neptunewastemanagement.com/neptunesmoke-evacuator/. Accessed April 20, 2020.

12. DeGirolamo KM, Courtemanche DJ, Hill WD, Kennedy A, Skarsgard ED. Use of safety scalpels and other safety practices to reduce sharps injury in the operating room: what is the evidence? Can $J$ Surg.2013;56(4):263-269.

Tables:

Table 1: Surgical case details and aerosol generating instrument use.

\begin{tabular}{llllll}
\hline Case & Diagnosis & Surgery & Microdebrider & Bone Drilling & Caut \\
\hline 1 & Osteomyelitis and sinusitis & ESS & No & No & Yes \\
2 & Adenoid Cystic Carcinoma & Transpterygoid nasopharyngectomy & Yes & Yes & Yes \\
3 & NK T-cell Lymphoma & ESS and transoral resection & No & No & No \\
4 & Pituitary Adenoma & ESS, Transphenoidal pituitary resection & Yes & Yes & Yes \\
\hline
\end{tabular}

ESS - endoscopic sinus surgery; NK - natural killer

Figure Legends 


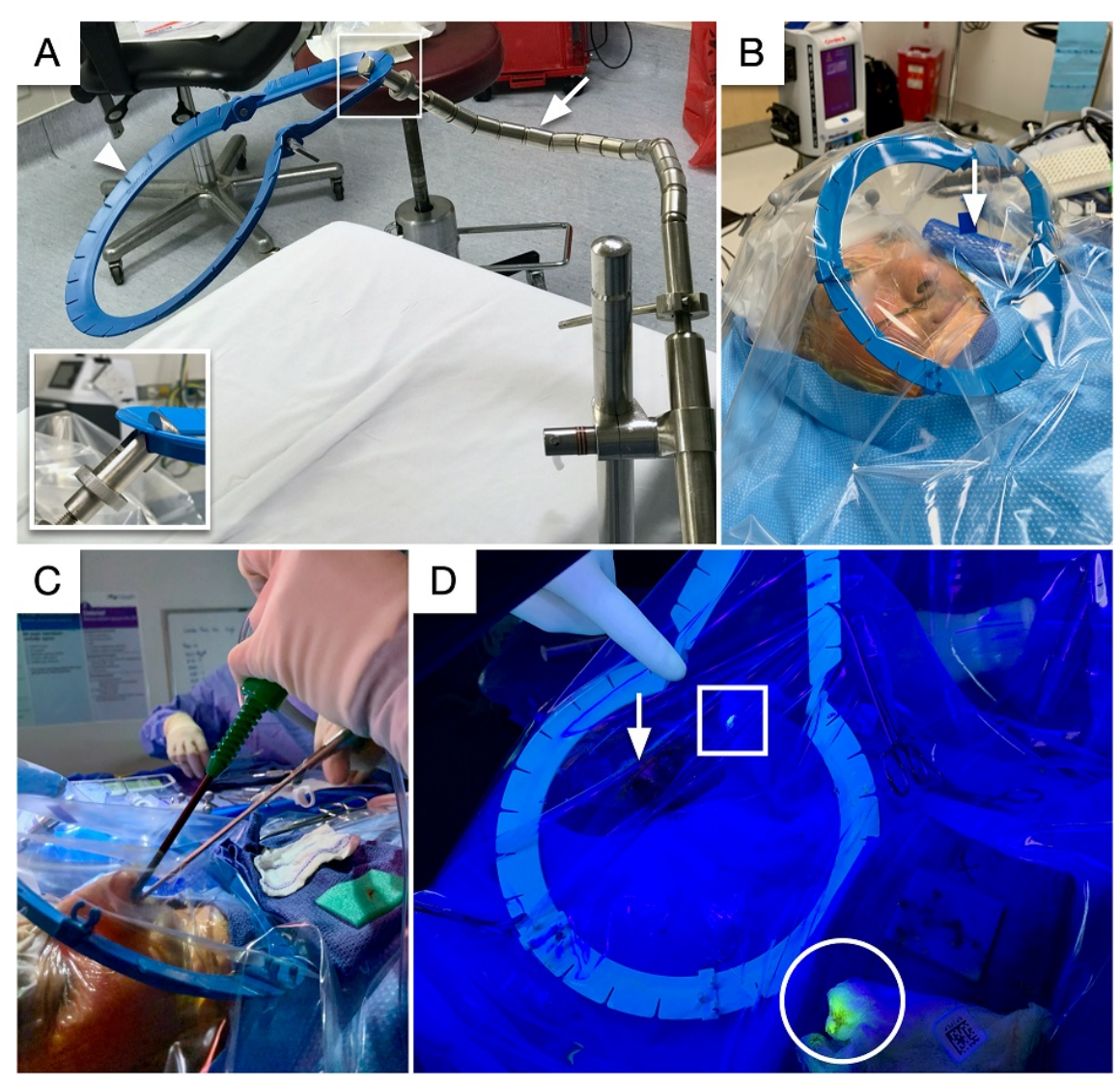

Figure 1: This is a caption

Figure 1: The negative-pressure otolaryngology viral isolation drape (NOVID) setup. (A) The apparatus consists of a laparoscopic Bookwalter holder (arrow) and a lone star ring retractor (arrow-head), with a close up view of the attachment point (square inset); (B) HEPA filter (arrow) connected to suction beneath the drape; (C) endoscope and instrument insertion through fenestrations in the drape; (D) fluorescein-laden droplets at the mouth of the suction device (square) and gauze used to clean the scope (circle), the patient's nose (arrow) for reference. 

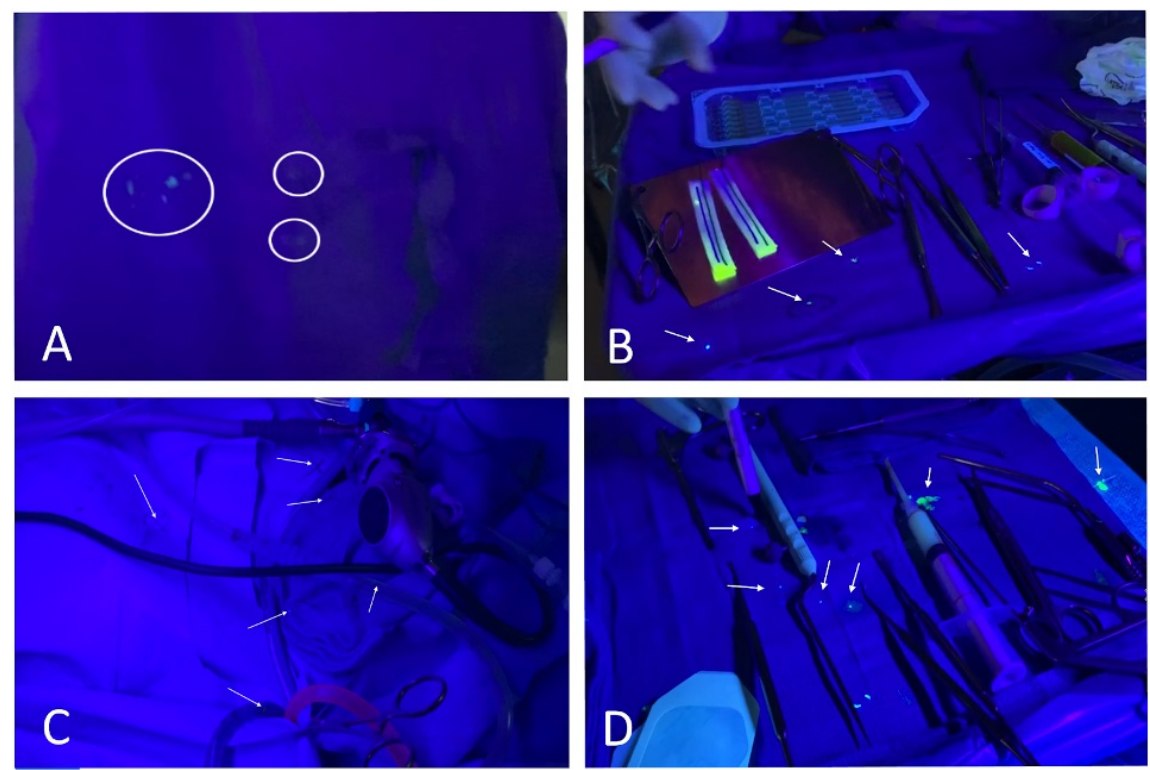

Figure 2: This is a caption

Figure 2: Postoperative black light exams. Panel A and B from Case 2. (A) Demonstration of gross contamination of the nurse's gown on the abdomen, after directly handling epinephrine-soaked cottonoids. (B) Multiple $>5 \mathrm{~mm}$ droplets on the instrument table. Panels C-F from Case 4. (C) Multiple small droplets $<1 \mathrm{~mm}$ on the surgical field at chest level. (D) Instrument table with large drops $>5 \mathrm{~mm}$. 